\title{
GALAXY EVOLUTION PROBED BY MID-INFRARED DEEP SURVEYS
}

\author{
Y. TANIGUCHI \\ Astronomical Institute, Tohoku University \\ Aramaki, Aoba, Sendai 980-77, JAPAN
}

\begin{abstract}
We discuss the formation and evolution of galaxies in view of recent mid-infrared deep surveys made using Infrared Space Observatory. A special interest is addressed to finding reddened massive populations at high redshifts.
\end{abstract}

\section{ARE THERE ANY HIDDEN POPULATIONS AT HIGH REDSHIFTS?}

When and how did galaxies form ? There may be two alternative formation scenarios for the formation of galaxies; one is the dissipative collapse of a massive protogalactic cloud and the other is successive mergers among subgalactic clumps (see Figure 1). There are several lines of evidence for massive gaseous systems at high redshifts (Ohta et al. 1996; Omont et al. $1996 \mathrm{a}, 1996 \mathrm{~b}$ ). Star formation history of massive (disk) galaxies at redshifts $z<2$ have been also investigated extensively based on a nearly complete galaxy sample down to $K=20, I=23$, and $B=24.5$ (Cowie et al. 1996, 1997). On the other hand, star-forming objects with subgalactic scales (e.g., $\sim 1 \mathrm{kpc}$ ) have been also observed (e.g., Lanzetta et al. 1996; Pascarelle et al. 1996; Trager et al. 1997). Therefore, even now, it is still difficult to give a definite answer to the above question (see for recent reviews, Fukugita et al. 1996; Ellis 1997).

Since typical galaxies in the local universe are very old (more than 10 billion years), we have to probe the high redshift universe in order to investigate their infant phase. Recent deep survey observations in the optical have been powerful for detecting very faint sources in the high- $z$ universe. Candidates of forming galaxies which have been found for these years are 
summarized in Table 1. Although these high- $z$ galaxies seem indeed forming galaxies, we still have a question; Have we found all the $z>3$ galaxies ? Are we missing any of the population by virtue of selection effects? What are the prospects for finding higher redshift sources? (Ellis 1996). The observed surface density of such high- $z$ star forming galaxies may be lower than the value expected from the local density of luminous galaxies (e.g., Lanzetta et al. 1996; Steidel et al. 1996b; see, however, Lowenthal et al. 1997) and it is possible that we are missing classes of objects with substantial reddening and have not yet sampled fully the high- $z$ counterparts of typical nearby galaxies (Franceschini et al. 1994, 1997; Mazzei and De Zotti 1996; Cimatti et al. 1997).

TABLE 1. Some important objects at high redshifts

\begin{tabular}{|c|c|c|}
\hline Objects & Redshifts & References \\
\hline$R$-drop Lyman break galaxies & $\sim 7$ & Lanzetta et al. 1996, Nat, 381, 759 \\
\hline Lensed, high- $z$ galaxies & 4.92 & Franx et al. 1997, ApJ, 486, L75 \\
\hline Ly $\alpha$ emission-line galaxies & 4.55 & Hu \& McMahon 1996, Nat, 382, 231 \\
\hline$U$-drop Lyman break galaxies & $\sim 3-3.5$ & $\begin{array}{l}\text { Steidel et al. 1996b, ApJ, 462, L17 } \\
\text { Steidel et al. 1996a, AJ, 112, 352 }\end{array}$ \\
\hline$\cdot$ & & Lowenthal et al. 1997, ApJ, 481,673 \\
\hline Young, emission-line galaxies & $\sim 2.5-2.7$ & $\begin{array}{l}\text { Yee et al. 1996, AJ, } 111,1783 \\
\text { Malkan et al. 1995, ApJ, } 448, \text { L5 }\end{array}$ \\
\hline High- $z$, Lyman absorption system & 3.15 & Djorgovski et al. 1996, Nat, 382, 234 \\
\hline High- $z, \mathrm{Mg}$ II absorption system & 4.38 & Elston et al. 1996, ApJ, 456, L13 \\
\hline $\begin{array}{l}\text { The most distant quasar known } \\
\text { The most distant radio galaxy known }\end{array}$ & $\begin{array}{c}4.897 \\
4.41\end{array}$ & $\begin{array}{l}\text { Schneider et al. } 1991, \mathrm{AJ}, 102,837 \\
\text { Rawlings et al. } 1996, \mathrm{Nat}, 383,502\end{array}$ \\
\hline
\end{tabular}

\section{FINDING HIDDEN POPULATIONS}

Since there are many massive elliptical galaxies in the local universe, it is very important to find their high- $z$ counterparts. If the massive elliptical galaxies (particularly in clusters of galaxies) underwent vigorous starburst in their forming phase (e.g., Ellis et al. 1997 and references therein), they would have been already found because their Ly $\alpha$ emission could be strong enough to be detected by existing searches (Partridge and Peebles 1967; Pahre and Djorgovski 1995; Thompson et al. 1996; see for a review Pritchet 1994). These negative results suggest either that they may be hidden by dust grains or that the star formation activity in them may be 


\section{Formation \& Evolution of Galaxies}

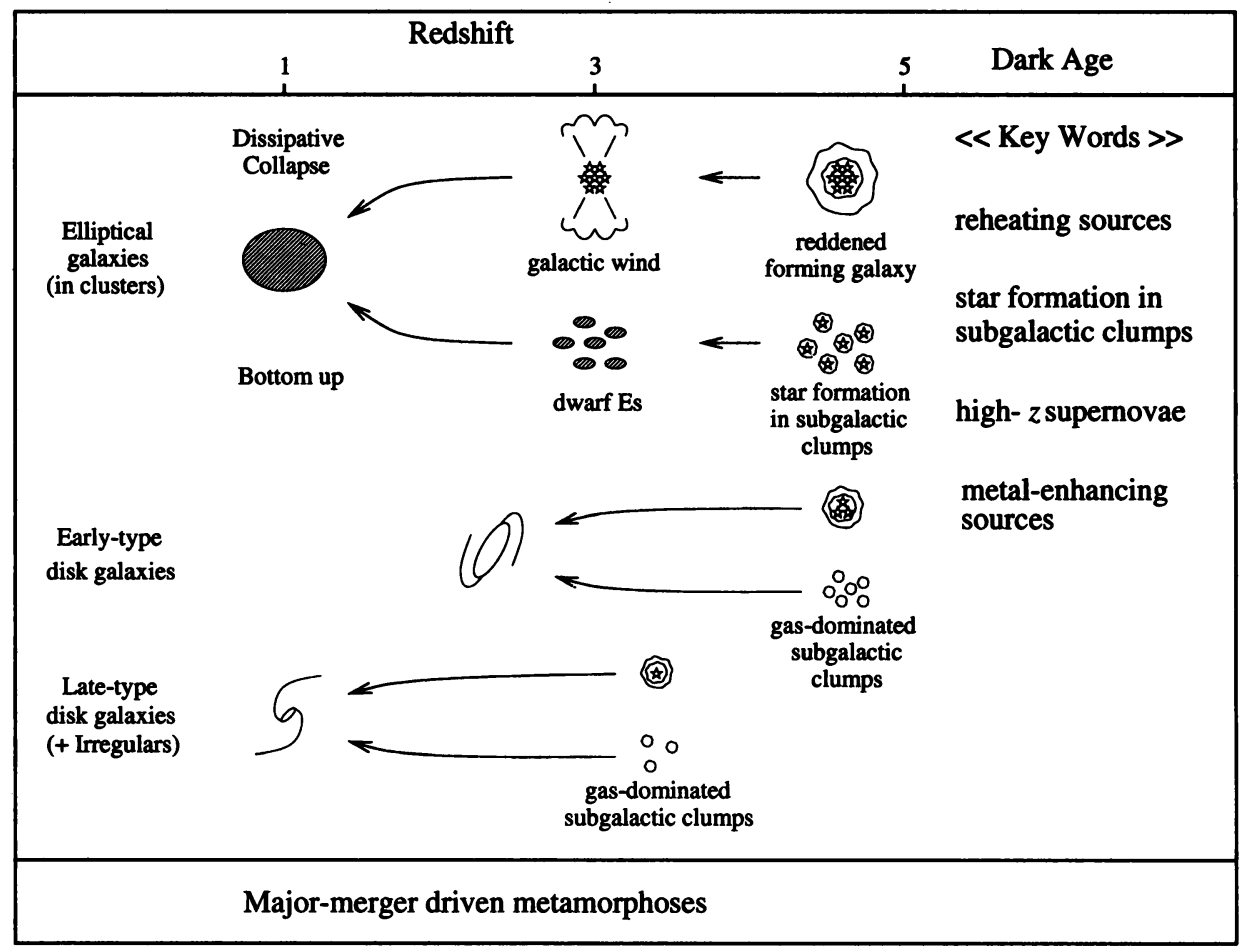

Figure 1. Two alternative scenarios for galaxy formation (either dissipative collapse of a massive gaseous system or successive mergers among clumps) are schematically shown for each type of galaxies; ellipticals, early-type spirals, and late-type spirals. Another important process to form present-day galaxies may be major mergers (see the last row). Some important key words at very high redshifts $(z>5)$ are also shown in the right-hand panel (e.g., Miralda-Escudé and Rees 1997; Gnedin and Ostriker 1997 and references therein).

more quiescent than what we thought. According to the standard galactic wind model for elliptical galaxies (Arimoto and Yoshii 1987; Kodama and Arimoto 1997), the forming phase before the galactic winds (the first $\sim 1 \mathrm{Gyr}$ ) could be heavily obscured by abundant gas and dust inside the system. Massive gaseous matter $\left(\sim 10^{10-11} M_{\odot}\right)$ has been indeed found in host galaxies of high- $z$ quasars and powerful radio galaxies (Ohta et al. 1996; Omont et al. 1996a, 1996b). Such powerful active galactic nuclei are usually associated with nuclei of massive (elliptical) galaxies in the nearby universe. Thus it is expected that the high- $z$ quasar hosts are intimately related to the formation of massive galaxies. If the quasar engine itself is reddened heavily by gas and dust in its host, we could miss such a class of objects in the existing optical deep surveys. Therefore, it is worth searching 
for such reddened populations at high redshifts.

Let us consider how to find them (Sato 1994). Since the nearby, well evolved galaxies consist of old low-mass stars, it is important to find such galaxies at high redshifts. These stars emit their energy from the optical to near infrared (NIR) mostly, implying that they are more luminous in the NIR and MIR (mid-infrared) than in the optical if they were present at high redshifts. MIR searches have another advantage because the effect of reddening is less than that in NIR. Infrared Space Observatory (ISO, Kessler et al. 1996) allows us to perform such a deep survey since ISOCAM (the infrared camera on-board ISO) has a very high sensitivity in the MIR (Cesarsky et al. 1996). Following a dissipative collapse model for elliptical galaxies (Larson 1974), Meier (1976) calculated the spectral energy distribution (SED) of a forming elliptical galaxy [Model B in Larson (1974)]. In Figure 2, we show SEDs of a primeval galaxy with luminosity of $10^{12.5} L_{\odot}$ at a redshift 3 as a function of extinction where we adopt the standard Friedman Universe with a Hubble constant $H_{0}=75 \mathrm{~km} \mathrm{~s}^{-1} \mathrm{Mpc}^{-1}$ and a deceleration parameter $q_{0}=0.5$. This figure shows that if we perform a very deep survey in the mid-infrared (MIR) using ISO, it is possible to detect such high- $z$, forming elliptical galaxies (see Figure 3). In particular, the LW2 filter band $\left(\lambda_{\text {center }}=6.75 \mu \mathrm{m}\right)$ provides the best observational window.

\section{MID-INFRARED DEEP SURVEYS}

A couple of very deep MIR surveys have been made using ISOCAM (RowanRobinson et al. 1997; Taniguchi et al. 1997). A brief summary of both surveys is given in Table 2 (Taniguchi 1997). Rowan-Robinson et al. (1997) made the ISOCAM observations of the Hubble Deep Field (HDF, Williams et al. 1996) at 7 and $15 \mu \mathrm{m}$. Combined with the optical multi-color photometry, these MIR data have been used to investigate the star formation properties of galaxies. Rowan-Robinson et al. (1997) modeled the spectral energy distribution of $14 \mathrm{HDF}$ galaxies with redshifts from 0.3 to 1.6 and found that 10 galaxies show the strong MIR excess, providing evidence for intense starbursts in these galaxies ${ }^{1}$ (see also Oliver 1997).

Another MIR deep survey program has been made by Taniguchi et al. (1997) whose main aim is just addressed to the discovery of heavily reddened populations at high redshifts. Their target field is selected in the Lockman HI hole because the Galactic HI column density, $\sim 4 \times 10^{19} \mathrm{~cm}^{-2}$, is the lowest value found over the entire sky, providing the best cosmological window for any extragalactic deep survey observations (Jahoda, Lockman,

\footnotetext{
${ }^{1}$ Recently Aussel et al. (1997) analyzed the ISO-HDF data independently using the PRETI (Pattern REcognition Technique for ISOCAM) method developed at Saclay.
} 


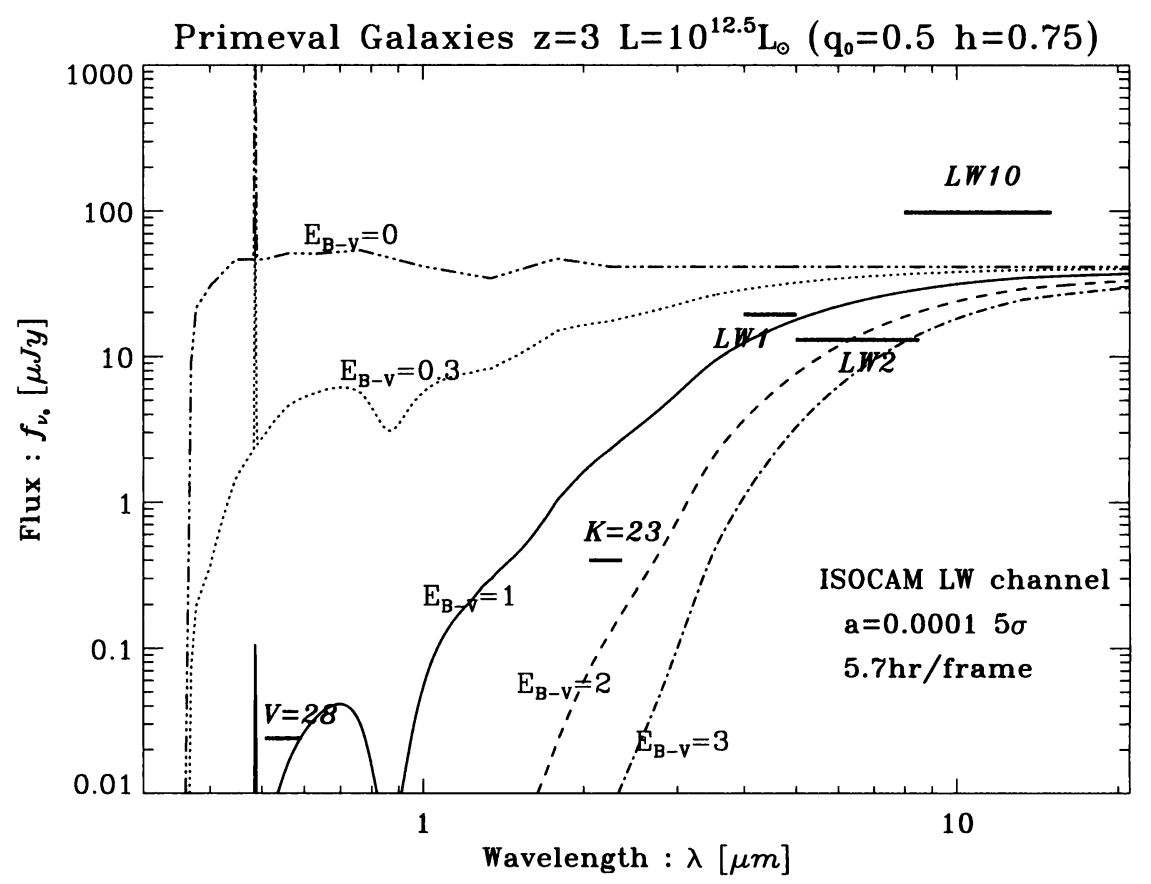

Figure 2. SEDs of a forming elliptical galaxy as a function of extinction $\left(E_{B-V}=0,0.3\right.$, $1,2$, and 3$)$. We show $5 \sigma$ detection limits for three LW channels available for ISOCAM $\left(L W 1 ; \lambda_{\text {center }}=4.5 \mu \mathrm{m}, L W 2 ; \lambda_{\text {center }}=6.75 \mu \mathrm{m}\right.$, and $\left.L W 10 ; \lambda_{\text {center }}=11.5 \mu \mathrm{m}\right)$ in the case of 5.7 hours integration. The flat fielding error $(a)$ is assumed to be 0.0001 . The detection limits are also shown for $V=28$ and $K=23$.

and McCammon 1990). Their deep image at $7 \mu \mathrm{m}$ is shown in Figure 4. The background fluctuation of the central part of this mage is $0.09 \mu \mathrm{Jy} \mathrm{arcsec}-2$, corresponding to a $3 \sigma$ detection limit of $\simeq 9.7 \mu \mathrm{Jy} \mathrm{beam}^{-1}$ (the beam size $\left.=6^{\prime \prime} \times 6^{\prime \prime}\right)$. This limit is very close to the expected value in the ISOCAM observers manual. Thus their deep imaging observations have confirmed the very high in-flight performance of ISOCAM.

Although they detected 55 sources ( 27 sources are above $5 \sigma_{\text {rms }}$ ), they described the nature of only 15 sources with quite high significance (see Figure 4). Among the 15 sources, one source is not observed in their NIR observations because the position angle of the LW2 image is shifted by $\sim 30^{\circ}$ from those of the NIR images. Among the remaining 14 sources, twelve sources have NIR counterparts. The observed flux ratios between LW2 and $K^{\prime}\left[R \equiv f_{\nu}(\mathrm{LW} 2) / f_{\nu}\left(K^{\prime}\right)\right]$ scatter from $\sim 0.2$ to 7.4 although most of them are less than $\sim 1$. Since typical late-type dwarf stars show no MIR excess in their rest-frame spectra, the objects with smaller ratios (e.g., $R<0.5)$ may be Galactic stars or early type galaxies with moderate 


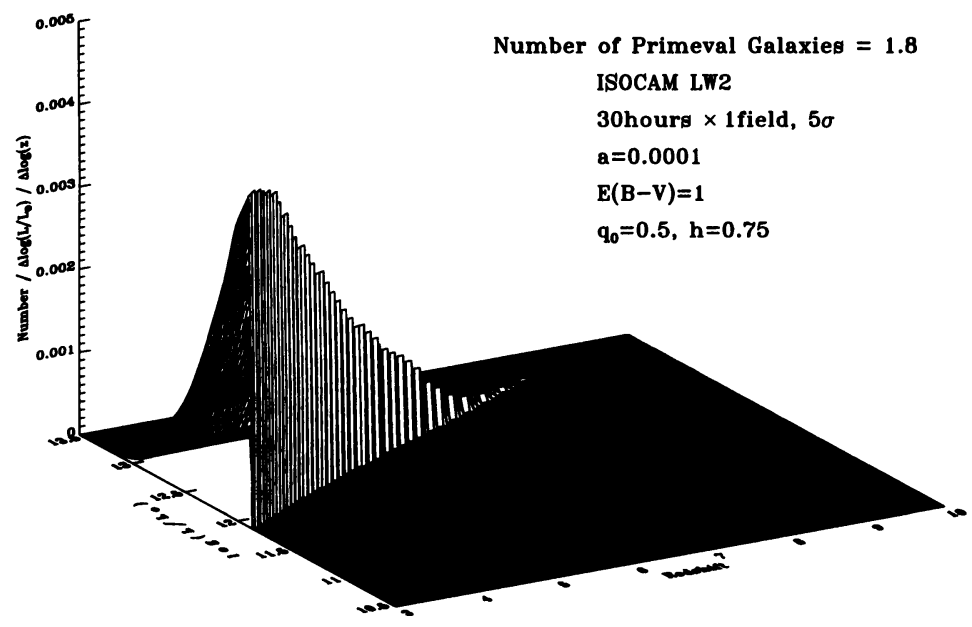

Figure 3. Physical parameters (luminosities and redshifts) of galaxies detected by a very deep survey at $7 \mu \mathrm{m}$ using ISOCAM (Sato 1994). Here we adopt an integration time of 30 hours for a $3^{\prime} \times 3^{\prime}$ field. The $K$-band luminosity function for local galaxies derived by Franceschini et al. (1991) is assumed.

TABLE 2. Very deep surveys in the mid infrared

\begin{tabular}{lcccc}
\hline & \multicolumn{2}{c}{ Rowan-Robinson et al. } & \multicolumn{2}{c}{ Taniguchi/Cowie et al. } \\
\hline Field & & HDF & LH-NW & SSA 13 \\
Filter & LW2 & LW3 & LW2 & LW2 \\
$\lambda_{\text {center }}(\mu \mathrm{m})$ & 6.75 & 14.5 & 6.75 & 6.75 \\
TDT $^{a}($ hours $)$ & $6.5^{b}$ & 6 & 13.4 & 18 \\
PFOV $^{b}(\operatorname{arcsec})$ & 3 & 6 & 6 & 6 \\
Area $\left._{(\operatorname{arcmin}}{ }^{b}\right)$ & 5 & 15 & 9 & 9 \\
$f\left(5 \sigma_{\text {rms }}\right)(\mu \mathrm{Jy})$ & $40^{d}$ & $200^{d}$ & 16 & $-{ }^{e}$ \\
No. of sources & $27^{d}$ & $22^{d}$ & 55 & $-{ }^{e}$ \\
\hline
\end{tabular}

a Target dedicated time.

${ }^{b}$ Another 6.5-hour integration has been obtained recently (Rowan-Robinson 1997, private communication).

${ }^{c}$ Pixel field of view.

${ }^{d}$ Goldschmidt et al. (1997). See also Aussel et al. (1997).

e Not yet reduced.

redshifts. On the other hand, starburst galaxies and active galactic nuclei 


\section{ISOCAM $7 \mu \mathrm{m}$ image (halftone) and $\mathrm{H}+\mathrm{K}$ image (contour)}

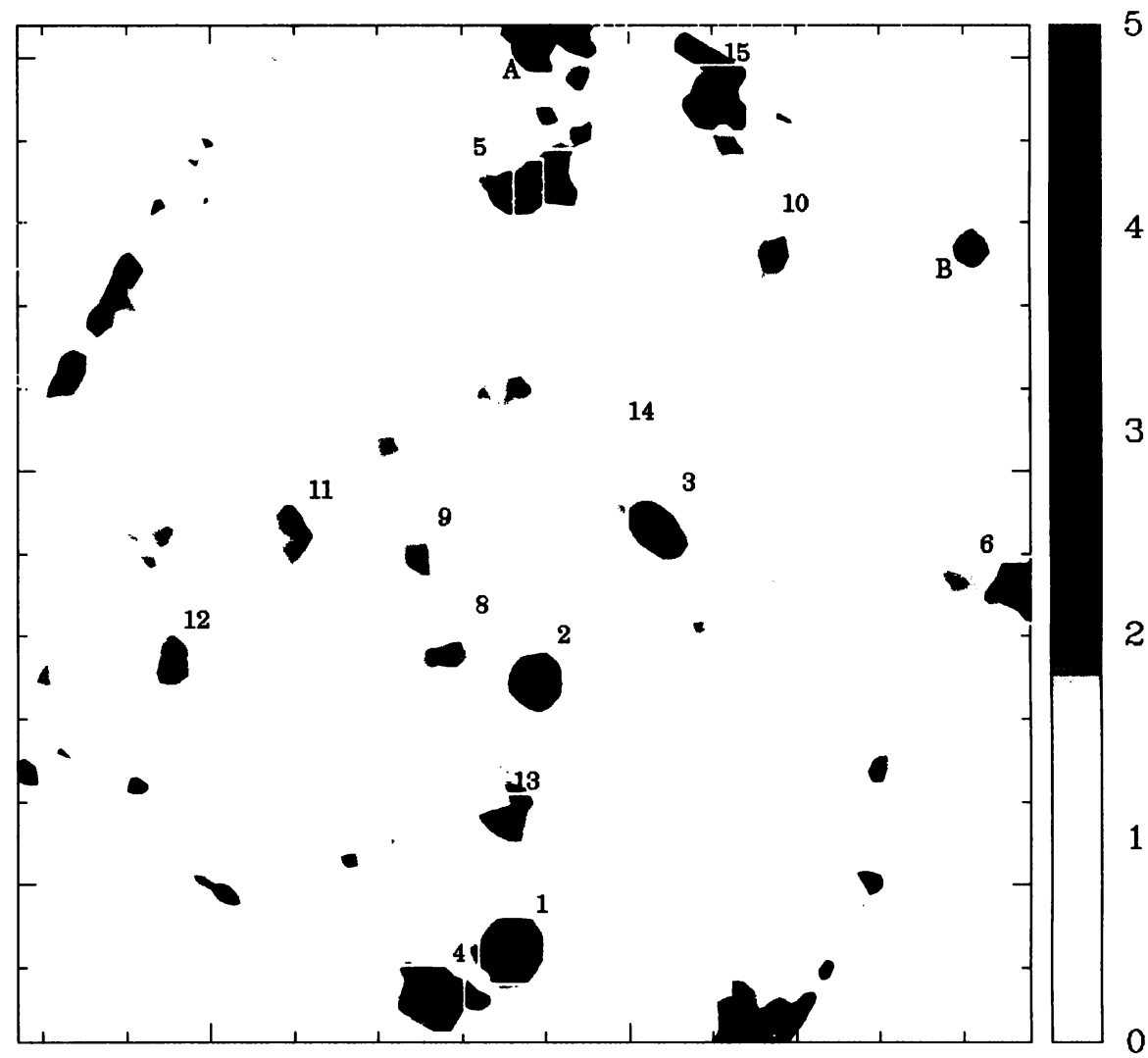

Figure 4. The $7 \mu \mathrm{m}$ mosaiced image of a $3^{\prime} \times 3^{\prime}$ area in the Lockman HI hole (halftone; darker is brighter) obtained by Taniguchi et al. (1997). The contours show the NIR $(H K)$ image taken with the University of Hawaii $2.2 \mathrm{~m}$ telescope. Although the seeing size in the NIR image is very good (FWHM $\simeq 0.8$ arcsec), the image is blurred in order to make the comparison between the LW2 and the NIR images easier. The two sources labeled by $A$ and $B$ have NIR counterparts and thus they may be real sources though their $7 \mu \mathrm{m}$ fluxes are below $5 \sigma$. North is up and east is left.

usually show a MIR excess (e.g., Rowan-Robinson et al. 1997). Therefore the objects with the larger ratios may be such galaxies with moderate redshifts. Most of the sources have $K$ magnitudes between 16 and 20 and thus they may have properties similar to the faint NIR galaxies studied by Cowie et al. (1996). If this is the case, the majority of these $7 \mu \mathrm{m}$ sources may be galaxies or active galactic nuclei with $0.2<z<2$ (Cowie et al. 1996).

It is noted that two objects (Nos. 9 and 12 in Figure 4) have no apparent 
NIR counterpart. Though a faint NIR source is located near each $7 \mu \mathrm{m}$ object, the center position is significantly different $\left(\sim 4^{\prime \prime}\right)$ between the LW2 and NIR images. Even if these NIR sources were the counterparts, these two objects have very large $R$ values, suggesting heavy reddening. Therefore, we consider that they are heavily reddened populations which have not been detected by existing optical and NIR deep surveys.

\section{PROSPECTS}

The most important conclusion is that ISO enabled us to perform the source detection at a level of $\sim 10 \mu \mathrm{Jy}$ at $7 \mu \mathrm{m}$ (Rowan-Robinson et al. 1997; Taniguchi et al. 1997). These MIR deep survey projects have provided a new frontier in the study of star formation history of galaxies at high redshifts. We hope that such MIR deep surveys will be conducted using nextgeneration infrared space telescope facilities like SOFIA (Stratospheric Observatory for Infrared Astronomy which will be operated in 2001; Erickson 1997) and IRIS (Infrared Imaging Surveyor which will be launched in 2003; Matsumoto 1997).

One problem remains; how to follow up the $7 \mu \mathrm{m}$ sources found by Taniguchi et al. (1997). These objects cannot be seen in the optical and thus we cannot do optical spectroscopy of them. If these objects are really heavily reddened population at high redshifts, they would be very bright at far infrared in their rest frame (e.g., Eales and Edmunds 1996). Since their redshifts are expected to be $z>3$, they may be bright at submillimeter regime in the observed frame (their expected fluxes are a few Jy at 100 $\mathrm{GHz}$ though they depend on their redshifts). Therefore, the existing radio interferometer facilities will be able to detect them.

I would like to thank all the staff of ISO, in particular Martin Kessler, Catherine Cesarsky, and David Elbaz for their useful comments and encouragement during the course of this work. I would like to thank Richard Ellis, Michael Rowan-Robinson, Seb Oliver, Nobuo Arimoto, Yuzuru Yoshii, Toru Yamada, and Toshinobu Takagi for useful comments. Finally I thank my colleagues, Len Cowie, Dave Sanders, Bob Joseph, Gareth Wynn-Williams, Ken Chambers, H. Okuda, K. Kawara, T. Matsumoto, K. Wakamatsu, Y. Sofue, H. Matsuhara, and Yasu Sato.

\section{References}

Arimoto, N., and Yoshii, Y. (1987) Chemical and Photometric Properties of a Galactic Wind Model for Elliptical galaxies, $A \mathscr{B} A, \mathbf{1 7 3}, 23$

Aussel, H., Elbaz, D., Starck, J.L., and Cesarsky C.J. (1997) Observation of the Hubble Deep Field with ISOCAM: Application of the PRETI Method, in Extragalactic Astronomy in the Infrared, eds. G. Mamon, T. Thuan, J. TranhVan (Gif-sur-Yvette: 
Ed. Frontieres), in press

Cesarsky, C.J., Abergel, A., Agnese, P., et al. (1996) ISOCAM in Flight, $A \& A$, 315, L32

Cimatti, A., Bianchi, S., Ferrara, A., and Giovanardi, C. (1997) On the Dust Extinction in High- $z$ Galaxies and the Case of Extremely Red Objects, MNRAS, 290, L43

Cowie, L.L., Hu, E.M., Songaila, A., and Egami, E. (1997) The Evolution of the Distribution of Star Formation Rates in Galaxies, $A p J, 481$, L9

Cowie, L.L., Songaila, A., Hu, E.M., and Cohen, J.G. (1996) New Insight on Galaxy Formation and Evolution From Keck Spectroscopy of the Hawaii Deep Fields, $A J$, 112, 839

Djorgovski, S.G., Pahre, M.A., Bechtold, J., and Elston, R. (1996) Identification of a Galaxy Responsible for a High-Redshift Lyman- $\alpha$ Absorption System, Nature, 382, 234

Eales, S.A., and Edmunds, M.G. (1996) The Implications of Large Dust Masses at High Redshifts: a First Look at Galactic Evolution in the Submillimeter Waveband, $M N$ $R A S, 280,1167$

Ellis, R.S. (1996) Introduction, in the 37th Herstmonceux Conf. The Hubble Space Telescope and the High Redshift Universe, eds. N. R. Tanvir, A. Aragon-Salamanca, J. V. Wall, 1

Ellis, R.S. (1997) Faint Blue Galaxies, $A R A$ \& $A$, 35, in press

Ellis, R.S., et al. (1997) The Homogeneity of Spheroidal Populations in Distant Clusters, ApJ, 483, 582

Elston, R., Bechtold, J., Hill, G.J., and Ge, J. (1996) A Redshift 4.38 Mg II Absorber toward BR 1202-0725, ApJ, 456, L13

Erickson, E.F. (1997) Sofia: The Next Airborne Observatory, Diffuse Infrared Radiation and the IRTS, ASP Conf. Ser., 124, eds. H. Okuda, T. Matsumoto, and T.L. Roelling, 415

Franceschini, A., De Zotti, G., Toffolatti, L. Mazzei, P., and Danese, L. (1991) Galaxy Counts and Contributions to the Background Radiation from 1 micron to $1000 \mathrm{mi}-$ crons, $A \& B S, 89,285$

Franceschini, A., Mazzei, P., De Zotti, G., and Danese, L. (1994) Luminosity Evolution and Dust Effects in Distant Galaxies: Implications for the Observability of the Early Evolutionary Phases, $A p J, 427,140$

Franceschini, A., Silva, L., Granato, G.L., Bressan, A., and Danese, L. (1997) Old Massive Ellipticals and S0s in the Hubble Deep Field Vanish from View at $z>1$.3: Possible Solutions of the Enigma, $A p J$, submitted (ASTRO-PH/9707064)

Franx, M., Illingworth, G.D., Kelson, D.D., van Dokkum, P.G., and Tram, K.-V. (1997) A Pair of Lensed Galaxies at $z=4.92$ in the Field of CL 1358+62, ApJ, 486, L75

Fukugita M., Hogan C.J., Peebles P.J.E. (1996) History of Galaxies, Nature, 381, 489

Gnedin, N.Y., and Ostriker, J.P. (1997) Reioniza+ ${ }^{\circ}$ on of the Universe and the Early Production of Metals, ApJ, 486, 581

Goldschmidt, P., Oliver, S., Serjeant, S., et al. (1997) Observations of the Hubble Deep Field with the Infrared Space Observatory - II. Source detection and photometry, MNRAS, 289, 465

Hu, E.M., and McMahon, R.G. (1996) Detection of Lyman- $\alpha$-emitting Galaxies at Redshift 4.55, Nature, 382, 231

Jahoda, K., Lockman, F.J., and McCammon, D. (1990) Galactic H I and the interstellar medium in Ursa Major, $A p J, 354,184$

Kessler, M.F., Steinz, J.A., Anderegg, M.E., et al. (1996) The Infrared Space Observatory (ISO) mission, $A \mathscr{B} A, \mathbf{3 1 5}, \mathrm{L} 27$

Kodama, T., \& Arimoto, N. (1997) Origin of the Colour-Magnitude Relation of Elliptical Galaxies, $A$ \& $A$, 320, 41

Lanzetta, K.M., Yahil, A., and Fernandes-Soto, A. (1996) Star-forming Galaxies at Very High Redshifts, Nature, 381, 759

Larson, R.B. (1974) Dynamical Models for the Formation and Evolution of Spherical Galaxies, MNRAS, 166, 585 
Lowenthal, J.D., Koo, D.C., Guzmán, R., et al. (1997) Keck Spectroscopy of Redshift $z \sim 3$ Galaxies in the Hubble Deep Field, $A p J, 481,673$

Malkan, M., Teplitz, H., and McLean, I. (1995) An Emission-line Protogalaxy Candidate at $z=2.5, A p J, 448, \mathrm{~L} 5$

Matsumoto, T. (1997) Infrared Imaging Surveyor: IRIS, Diffuse Infrared Radiation and the IRTS, ASP Conf. Ser., 124, eds. H. Okuda, T. Matsumoto, and T.L. Roelling, 454

Mazzei, P., and De Zotti, G. (1996) Dust in High-Redshift Radio Galaxies and the Early Evolution of Spheroidal Galaxies, MNRAS, 279, 535

Meier, D.L. (1976) The Optical Appearance of Model Primeval Galaxies, ApJ, 207, 343

Miralda-Escudé, J., and Rees, M. J. (1997) High Redshift Supernovae and the Metal-Poor Halo Stars: Signatures of the First Generation of Galaxies, ApJ, 478, L57

Ohta, K., Yamada, T., Nakanishi, K., Kohno, K., Akiyama, M., and Kawabe, R. (1996) Detection of Molecular Gas in the Quasar BR1202-0725 at Redshift 4.69, Nature, 382,426

Oliver, S. (1997) Deep Surveys and Cosmology, Highlights in Astronomy, in press

Omont, A., McMahon, R.G., Cox, P., Kreseya, E., Bergeron, J., Pajot, F., and StorrieLombardi, L.J. (1996a) Continuum Millimeter Observations of High-Redshift RadioQuiet QSOs, $A \& A, \mathbf{3 1 5}, 1$

Omont, A., Petitjean, P., Guilloteus, S., McMahon, R.G., Solomon, P.M., and Pecontal, E. (1996b) Molecular Gas and Dust Around a Radio-Quiet Quasar at Redshift 4.69 Nature, 382, 428

Pahre, M.A., and Djorgovski, S.D. (1995) A Near-Infrared Search for Line Emission from Protogalaxies Using the W.M. Keck Telescope, ApJ, 449, L1

Partridge, R.B., and Peebles, P.J.E. (1967) Are Young Galaxies Visible ? ApJ, 147, 868

Pascarelle, S.M., Windhorst, R.A., Keel, W.C., and Odewahn, S.C. (1996) Sub-galactic Clumps at a Redshift of 2.39 and Implications for Galaxy Formation, Nature, 383, 45

Pritchet, C.J. (1994) The Search for Primeval Galaxies, PASP, 106, 1052

Rawlings, S., Lacy, M., Blundell, M., Eales, S.A., Bunker, A.J., and Garrington, S.T. (1996) A Radio Galaxy at Redshift 4.41 Nature, 383, 502

Rowan-Robinson, M., Mann, R.G., Oliver, S.J., et al. (1997) Observations of the Hubble Deep Field with the Infrared Space Observatory - V. Spectral energy distributions, starburst models and star formation history, MNRAS, 289, 490

Sato, Y. (1994) Feasibility Study of a Search For Primeval Galaxies by ISO, Master Thesis (Tohoku University)

Schneider, D.P., Schmidt, M., and Gunn, J.E. (1991) PC 1247 + 3406 - An Optically Selected Quasar with a Redshift of 4.897, $A J, 102,837$

Steidel, C.S., Giavalisco, M., Dickinson, M., and Adelberger, K.L. (1996a) Spectroscopy of Lyman Break Galaxies in the Hubble Deep Field, $A J, 112,352$

Steidel, C.S., Giavalisco, M., Pettini, M., Dickinson, M., and Adelberger, K.L. (1996b) Spectroscopic Confirmation of a Population of Normal Star-forming Galaxies at Redshifts $z>3, A p J, 462, \mathrm{~L} 17$

Taniguchi Y. (1997) Very Deep Surveys, Highlights in Astronomy, in press

Taniguchi, Y. Cowie, L.L. Sato, Y. et al. (1997) ISOCAM 7 micron Deep Survey of the Lockman Hole: A Mid-Infrared Search for Primeval Galaxies, $A \& A$, in press

Thompson, D., Mannucci, F., and Beckwith, S.V.W. (1996) A Narrowband Imaging Survey for High Redshift Galaxies in the Near Infrared, $A J, 112,1794$

Trager, S.C., Faber, S.M., Dressler, A., and Oemler, A. Jr. (1997) Galaxies at $z \simeq 4$ and the Formation of Population II, $A p J, 485,92$

Williams, R. E., Blacker, B., Dickinson, M., et al. (1996) The Hubble Deep Field: Observations, Data Reduction, and Galaxy Photometry, $A J, 112,1335$

Yee, H.K.C., Ellingson, E., Betchtold, J., Carlberg, R.G., and Culluandre, J.C. (1996) A Proto-galaxy Candidate at $z=2.7$ Discovered by its Young Stellar Population, $A J$, 111, 1783 